\title{
Décadrages Décadrages
}

cinéma, à travers champs Cinéma, à travers champs

10 | 2007

La trilogie de Dieu de João César Monteiro

\section{Entretien avec Mário Barroso}

Jérôme Bourquin et Raphaël Oesterlé

\section{(2) OpenEdition}

Journals

Édition électronique

URL : https://journals.openedition.org/decadrages/421

DOI : $10.4000 /$ decadrages.421

ISSN : 2297-5977

\section{Éditeur}

Association Décadrages

\section{Édition imprimée}

Date de publication : 10 avril 2007

Pagination : 62-67

ISBN : 978-2-9700582-5-0

ISSN : 2235-7823

Référence électronique

Jérôme Bourquin et Raphaël Oesterlé, «Entretien avec Mário Barroso », Décadrages [En ligne], 10 | 2007, mis en ligne le 10 décembre 2012, consulté le 03 avril 2022. URL : http://

journals.openedition.org/decadrages/421 ; DOI : https://doi.org/10.4000/decadrages.421

(B) Décadrages 


\section{Entretien avec Mário Barroso}

\section{par Jérôme Bourquin et Raphaël Oesterlé}

Intervenant après l'interruption du tournage de La comédie de Dieu (1995), Mário Barroso restera en charge de l'image sur les films de João César Monteiro - soit La comédie de Dieu, Le bassin de J. W. (1997), Les noces de Dieu (1998) et Va-et-vient (2003). Leur collaboration donnera lieu à un nouveau mode de filmage, dominé par le plan large et fixe, et où la quasi-absence d'éclairage artificiel permet l'accueil des contre-jours et des fausses teintes.

L'entretien s'est déroulé en deux temps: après une première partie traditionnelle, il s'est poursuivi devant les films de Monteiro, en pointant certaines séquences.

Votre collaboration marque une rupture stylistique forte dans le cinéma de Monteiro. Etait-ce le produit d'une volonté consciente de sa part, ou est-ce apparu petit à petit, au cours de votre travail?

C'est sûrement une décision de Monteiro à l'époque. Mais je ne sais pas si João l'avait exactement en tête. Il avait en tout cas une envie: c'était d'alléger tout le dispositif cinématographique des tournages; il n’aimait pas ça.

J'avais suivi de près la préparation du film qui allait devenir La comédie de Dieu. Je ne sais pas ce qui lui était passé par la tête, il a eu envie de tourner en scope. Et, je ne sais pas, du fait que cela s'appelle $L a$ comédie de Dieu, il y a peut-être eu un malentendu avec Dominique [Chappuis, le chef opérateur] : c'était une comédie, donc il fallait faire un truc avec beaucoup de lumière, etc. Et je pense que João César avait aussi envie de faire quelque chose de classique, avec des découpages, avec des champs-contrechamps, avec des choses comme cela. Et je suivais de loin cette lancée - ils ont quand même tourné trois semaines - et je savais que le film s'était arrêté parce qu'il avait trouvé cela insupportable. Il a tout arrêté, et le film n'a repris que sept ou huit mois après.

Et un jour, Joaquim Pinto, qui était le producteur, me téléphone et me demande: "Est-ce que... éventuellement...»- et moi j'étais très réticent, je dis: «Ecoute, ça me fait plaisir, mais je sais pas, il faut voir avec João". Et quand j'arrive à Lisbonne, on se donne rendez-vous dans un café, en plein été, il faisait très chaud, c'était très lumineux. Et la seule chose que João m’a demandé c'est: "Dis-moi, si je veux tourner sous ce porche...»-dehors, devant le café, tu voyais un porche qui était complètement noir, à l'intérieur - «... Si on veut tourner sous ce porche, est-ce que je pourrais tourner sans lumière?" Je réponds: «Ecoute, tu peux tourner sans lumière partout où tu veux, on peut tourner sans lumière en pleine nuit, à la campagne ou à la mer, seulement après, il faut qu'on soit conscient de l'image - ou de l'absence d'image qu'on va avoir». "Mais toi, tu accepterais de tourner làdessous sans lumière?» Je dis: "Je n'ai pas à accepter, je pars du principe que si tu veux tourner sans lumière, c'est que le résultat te convient». Et disons que le cas- 
ting d'examen a eu lieu comme cela: savoir si j'étais d'accord de tourner sous ce porche sans lumière.

Quand on a fait La comédie de Dieu, donc ce premier film (et ça c'est encore visible malgré tout), c'était éclairé - très peu éclairé - parce que j’ai un peu triché : notre accord, c'était de ne pas éclairer. Mais en revanche, il acceptait qu'on allume une lumière, par exemple s'il y avait une ampoule quelque part ou un néon. On arrivait, on allumait, ça pouvait passer... Donc, par exemple, j'avais remarqué en repérage un endroit où on pouvait mettre un néon ou quelque chose comme ça, et je disais au chef-éléctro: «Ecoute, passe la veille, remets le néon, parce que peut-être qu'on va pouvoir l'utiliser...» On ne se connaissait pas encore assez pour que je sois suffisamment rassuré par rapport à cela. Mais c'est un tout petit peu éclairé - et il n'était pas en désaccord, j'utilisais des projecteurs très petits...

Et c'est comme cela que la collaboration a commencé. Je me souviens, un jour, c'était vers la fin du film, on va tourner dans une piscine. Et moi j'avais repéré la piscine : il y avait beaucoup de lumière, il n'y avait pas du tout besoin d'éclairage. Et je me suis dit: on ne sait pas combien de temps on va rester, etc. Et alors comme il y avait une passerelle en haut de la piscine où on pouvait mettre des gens pour assister à des compétitions, ou quelque chose comme cela, je me suis dit: je vais mettre trois ou quatre projos là-haut, au cas où - le temps, ou la nuit, on ne sait jamais. Et donc je passais avec João et il ne disait jamais rien et après on est sorti et il me dit: "Mais, j’ai cru voir des projecteurs là-haut... Ils ne sont pas à nous, hein! Ils ne sont pas à nous?" J'ai répondu : "Comment cela, ils ne sont pas à nous?" "Non, c'est des choses qui sont à la piscine, non?» J'ai dit: "Non João, évidemment que c'est à nous». Il m'a demandé: "Mais pourquoi? Tu compte les allumer?" "Je ne sais pas, on verra si c'est nécessaire... » Et c'est là que j'ai compris... Il ne m'a rien dit, mais je l'ai senti très contrarié. Conclusion : je suis allé voir le chef-éléctro, je lui ai dit: "Ecoute, là on va boire un café avec João; il faut que quand on revient tourner, excuse-moi mais tout ce qu'on a mis là-haut, tu l'enlèves, tu le fais disparaître...»Et à partir de là, on a commencé à aller vers le sombre. C'était à la fin du film déjà : donc l'autre qu'on a fait, c'était pratiquement sans lumière. Et là, je n'ai même pas triché. Je lui disais : "Ecoute João, là on va filmer, mais je ne suis pas certain que ça va marcher». Dans le film qu'on a fait après, Le bassin de J. W., il n'y avait pas de lumière, et c'est là qu'on réussit à voir, on a des fausses teintes $\mathbf{1}$ superbes, des trucs sombres, et parfois tellement sombres qu'on n'y voit rien.

Donc la séquence de la Valse à mille temps, à ce moment-là il n'y avait plus que le soleil?

Il n'y avait que le soleil, on le voyait très bien: les nuages passaient tellement vite. Même sur un film normal... On n'aurait pas tourné ou on aurait tourné comme ça parce que c'était impossible de faire autre chose. Ce qui est très étonnant, c'est que par exemple il y a une jeune femme qui a fait une étude sur les fausses teintes au cinéma. Et c'est très drôle parce qu'elle était persuadée qu'on avait fait exprès, qu'on avait trafiqué, et, à l'école, ils avaient essayé de refaire des fausses teintes artificielles, et ils ne retrouvaient pas; c'est alors que je leur ai dit: «Il n'y a rien!» Et à un moment, on ne cherchait que ça. João, c'était les seules choses qu'il regardait.

Cela dit, quand on faisait ce genre de choses, on recevait des coups de fil du labo: "Ah, mais il y a des fausses teintes partout...» Même aujourd'hui, ça

1 Vincent Pinel, dans son Vocabulaire technique du cinéma (Nathan, Paris, 1996) en donne cette définition: "Fausse teinte / ÉCLAIRAGE. En extérieurs, variation de l'intensité et de la qualité de la lumière provoquée par le passage d'un nuage devant le soleil. / Abréviation: f. t. / Les fausses teintes sont la plaie du tournage en extérieurs et la cause de coûteuses pertes de temps. Il est en effet impossible de raccorder deux images successives, l'une prise en plein soleil, l'autre en soleil voilé, tant le rendu photographique (contraste, éclairement, couleur...) est différent. On est donc amené à attendre les conditions favorables ou... tout est à refaire avec le nouvel éclairage." (p. 160). 
m'enrage. Je dis: "Mais écoutez, vous ne remarquez pas que j'ai bien vu qu'il y avait des fausses teintes!» Alors tu me diras, c'est vrai que dans un film avec un découpage traditionnel, ça peut poser des problèmes les fausses teintes. Parce que si tu as des champscontrechamps, c'est des raccords difficiles.

Donc, il ne voulait pas que tu interviennes avec des projecteurs. Pourtant, il était à la recherche d'effets visuels. La fausse teinte, c'est de l'ordre de l'effet, mais c'est naturel. Comment est-ce que tu arrivais à composer avec cette volonté?

Avec João, il n’y avait pas de négociation. C’était quelqu'un qui avait un rapport au cinéma, et surtout au tournage, qui était très particulier: il ne supportait pas l'agitation d'un tournage, toutes les contraintes techniques. Et pourtant, il n'avait pas besoin de beaucoup de choses. En plus - tu trouves cela surtout chez les réalisateurs un peu moins sûrs d'eux, ils ne se sentent réalisateurs que s'ils connaissent la technique. Combien de réalisateurs me disent: "Mario, tu mets quoi, un 50 ?" «Ben, si tu veux. " "Non, mais qu'est-ce que tu penses?" "Ben, je pense rien, je ne sais pas, on met un objectif, on regarde, on voit, si ça te convient, on le garde. » Je faisais ça systématiquement avec João, qui voulait à tout prix ne pas se tromper. Il me demandait: "T’as mis un filtre?» Et je répondais: "Arrête, ne me parle pas de filtres, je déteste ça.» Et il adorait ça, qu'un technicien lui dise qu'il ne connaissait rien aux filtres et aux gélatines. Du coup, ça le rassurait. Par exemple, il me demandait: "Tu mets quelle pellicule?" Et il me disait son numéro. Chose que je suis incapable de savoir. Et il aimait dire le numéro, pour avoir le côté maîtrise technique du cinéma. Donc très souvent avec João, je faisais exactement l'inverse, je jouais à ne pas nommer les projecteurs. Je disais: "Mets-moi le tuyau, là, le truc»; et avec João, ça marchait très bien ce côté "on s'en fout de la technique». Donc, je ne négociais rien. Il me disait: "On peut tourner?» Je répondais : "Bien sûr, qu'on peut tourner». De temps en temps, je lui disais: «Là, ça risque d'être un peu trop sombre».
Il y avait aussi des problèmes de point qui étaient un peu délicats. João n'aimait pas trop qu'on tripote la caméra, ça venait du fait qu'avec Manoel de Oliveira j'avais fait tous ces films où on ne touchait pas la caméra. J'avais fait des films où j'étais directeur photo, cadreur et acteur principal: donc il fallait inventer une manière. Personne ne pouvait aller vers la caméra. Donc on faisait le cadre, on décidait à quel endroit il y avait le point, on bouchait l'œilleton, on lançait la caméra, je me mettais en place, et c'est tout. Et personne n'approchait la caméra, même pour faire le point, ce qui donne à certains films que j'ai faits avec Manoel un charme très particulier. D’ailleurs, après, il a commencé à le faire exprès. Mais au début, c'était une manière de faire ça sans que personne d'autre aille à la caméra ou ne touche à la caméra. João était un petit peu fasciné par ça.

\section{C'est toi qui cadrais?}

Oui, mais comme tu vois, l'écrasante majorité des plans sont fixes. Je cadrais de préférence sans cadrer. Le bassin de J. W., c'était un petit peu comme La comédie de Dieu, c'est-à-dire que les deux premiers jours de tournage ont été catastrophiques. On était encore dans un mode de tournage dit classique: on bougeait la caméra, etc. D’un côté, João était fasciné par les mouvements au cinéma, etc. Et en même temps, il ne maîtrisait pas ça: il disait: "Je veux un gros plan"; alors tu lui montrais un gros plan et... Il y avait une charrette tirée par un âne, "Alors qu'est-ce qu'on fait?" Si on est fixe, c'est tellement serré que la charrette va passer et tu ne vas même pas t'en rendre compte, qu'est-ce qu'il faut faire? "Alors, on peut suivre un petit peu» - "Ah non, c'est pas possible». Voilà. Et Le bassin de J. W. a été un truc très difficile. On a tout arrêté. Et il a décidé du jour au lendemain de changer complètement le film, et de faire à nouveau en plan fixe, il est entré comme comédien, etc. C'était une complication énorme. Mais normalement ce qui se faisait, c'est qu'on choisissait des focales plutôt larges, dans des plans fixes où il se mettait lui-même en scène, sans rien nous dire. Il ne nous disait pas ce qui allait 
se passer. Dans La comédie de Dieu, je bouge encore un peu de temps en temps, je le suis encore, mais ici, on déterminait l'espace et après il faisait ses ballets. Tout ça était un petit peu inattendu. Dans Le bassin de J. W., il y avait des moments très surprenants. Il pouvait arriver complètement nu rien que pour faire chier l'ingénieur du son qui avait eu la mauvaise idée d'essayer de lui mettre un micro-cravate - chose qu'il ne supportait pas.

Il cultivait un peu le conflit avec son équipe technique?

En permanence. Sincèrement, je pense que la seule personne avec qui il n'a jamais eu de conflit, c'est moi. Le seul conflit que j'ai eu a éclaté lors d'une projection de rushes de La comédie de Dieu. On avait tourné dans un bidonville, un truc assez sordide, qui s'était très mal passé et il a commencé par dire : «C'est quoi cette merde de son!» Le son, c'était celui qui était possible. Et après le plan a changé, et: "C'est quoi ça, c'est de l'image ça?» Alors là, je me suis levé et je suis sorti. Immédiatement, il est venu: "Attends attends, je veux pas...»Et c'est le seul moment où je me suis dit: "Ecoute, si tu trouves que l'image, c'est de la merde, tu appelles quelqu'un d'autre qui te fasse ce que tu aimes.» Et en plus il avait raison, l'image, c'était mauvais parce qu'on avait essayé d'éclairer. Avec peu de choses: j'avais essayé de mettre des mandarines dans son décor et du coup, c'était très bien éclairé, ça faisait très bien éclairé, ça faisait cinéma quoi, professionnel, et il n'aimait pas ça du tout.

Mais en général, sur La comédie de Dieu, il était très content. A mon avis, il découvrait quelque chose. Il n'était pas très sûr de ce qu'on était en train de faire, et chaque fois qu'on allait aux rushes, il était ravi, ça se voyait. Il y avait des choses que moi je n'aimais pas et qu'il aimait beaucoup. S'il avait eu de la patience, on aurait retourné deux ou trois séquences...

\section{Faisiez-vous beaucoup de prises?}

Il y avait très rarement deux prises. La deuxième prise ne se faisait que pour des raisons de comédie. A ce moment - et c'est moi qui lui ai apporté cette chose - tout le texte était écrit. Tout son texte était écrit sur d'énormes feuilles blanches qu'on disposait de façon à ce qu'il puisse les lire. Donc on pouvait répéter si vraiment il hésitait sur un mot, et même comme cela il disait: "Oui oui, mais ça je l'arrange après...»

Donc très peu de prises, mais est-ce qu'il y avait des répétitions durant la mise en place des plans?

Jamais. Il pouvait répéter tout seul, par exemple avec une jeune femme, mais c'était entre eux. On était très peu au courant.

Et comment se passait la direction des comédiens?

Il n'y avait pas de direction. La direction, c'était le placement dans le cadre. Ou c'était son énervement. Par exemple il y a un truc dans le bus dans Va-et-vient où un type joue le facho, et il était fou de rage, mais jamais il ne lui a dit un mot. Et je lui disais : «Demande lui de ne pas crier», mais il ne l'a jamais demandé. Il $\mathrm{y}$ avait deux types de comédiens: les jeunes femmes ne sont pas comédiennes, c'est des rencontres, et des comédiens dont il était très ami, donc qui savaient aller dans le sens qu'il aimait. Pour les jeunes filles, la direction ne se faisait pas sur le plateau, mais peut-être avant et entre eux.

Dans la mesure où c'est un cinéma très référentiel, avec beaucoup de citations textuelles ou musicales, est-ce que Monteiro avait des références visuelles précises, que ce soit en cinéma ou en peinture?

Sûrement, mais dans sa tête. On voyait beaucoup de films ensemble, de Ford entre autres, parfois pas jusqu'à la fin, et je me demandais toujours: «Est-ce qu'il va me demander...» Et non. Il y avait un film où il était fasciné par les mouvements de grues. Et c'était très étrange parce que ça lui plaisait, ces mouvements à l'intérieur de décors assez petits, et tu te disais : "Est-ce que il veut aller dans cette direction?" Et en fait non. 


\section{Séquence de la balance, au début de La comédie de Dieu}

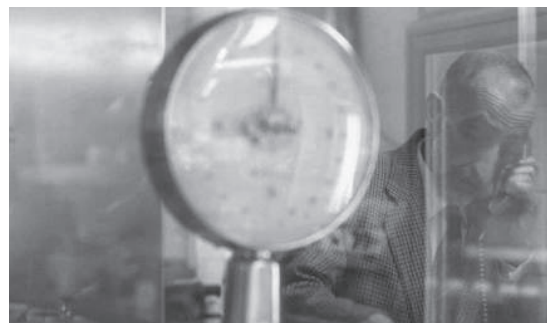

Là, il y a une première glace, une première vitre, ensuite ily a le reflet de la balance, les reflets métalliques derrière. Ces dispositifs, c'est vous deux qui les proposiez ou c'est lui qui venait, qui disait: "Je veux faire un plan avec des reflets à 3 degrés, à 4 degrés... ?»"

Non, non c'est un petit peu ce qu'on avait vu. On voyait qu'il y avait des reflets et on ne faisait rien pour les annuler. Mais on ne les a pas placés exprès. lances...

Et le fait qu'il y avait des reflets, les verres, les bril-

\section{Scène de dialogue entre Jean de Dieu et Rosarhino dans La comédie de Dieu}
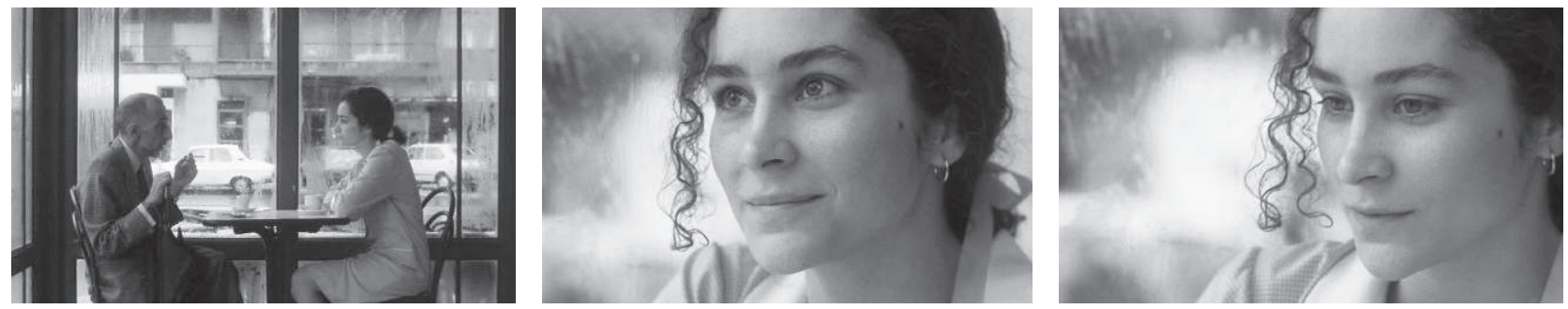

Là, c'était déjà mon truc: pratiquement sans rien, et j’adore ça.

Tu peux le commenter, le truc, l'expliciter en termes de rendu, le décrire?

Moi je pense qu'il y a un tout petit peu de lumière là, mais ce n'était pas absolument nécessaire, aujourd'hui je n'en mettrais pas. C'était encore une fois un accident, ce n'était pas voulu par le réalisateur ni par moi de faire pleuvoir à l'extérieur, c'est pas de la pluie de pompier, c'est de la vraie pluie, et c'est la chaleur à l'intérieur... Là où on est intervenu, c'est
C'était parce qu'ils étaient là et qu'on utilisait ce qu'il y avait.

Ce n'est pas toi qui demandais de replacer des...

Non, non. Attends, et en plus, normalement, un décor dans lequel on filmait, il restait tel qu'il était. Il disait: "Si j'ai choisi ce décor, c'est parce que je veux ça. Donc j'y touche pas.»A ce moment, il y avait peut-être un tout petit peu d'interférence de la part des décorateurs, mais sinon pratiquement partout, ce sont les décors tels qu'ils sont. qu'on a changé de table parce que le jeune accessoiriste, comme il y avait beaucoup de buée sur la vitrine, est allé donner un grand coup sur la vitrine, alors on a changé de table pour conserver la buée...

Tu vois: ça, c'est des cadres que j'aime beaucoup, mais qui après n'ont plus été possibles puisque si je faisais le cadre comme ça, elle se décadrerait en bougeant; là, j’ai donc été obligé de bouger un tout petit peu, mais c'était avec beaucoup de sobriété, il n'y a pas d'esbrouffe, c'est vraiment le plus sobre possible. 
Séquence du repas entre Jean de Dieu et la mère supérieure dans Les noces de Dieu

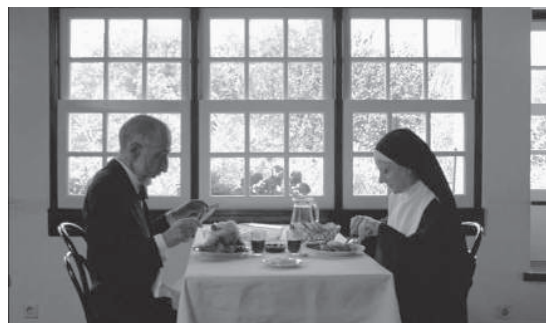

Voilà un plan qui pourrait être un plan qui n'est pas bon pour moi. Et en plus dans ce plan, j'utilise un peu de lumière... Parce que je pourrais parfaitement laisser tout ça dans le noir absolu, à l'intérieur.

Mais c'est pas beaucoup rattrapé, c'est assez silhouetté...

Oui, mais ça pourrait l'être beaucoup plus, parce qu'on n'a pas besoin de voir quoi que ce soit, la silhouette, c'était largement suffisant... Mais c'était pour voir un peu ce qu'ils avaient sur l'assiette... Quand le soleil disparaît, c'est mieux, mais quand il revient...

\section{Séquence du lendemain de la partie de poker dans Les noces de Dieu}

C'est peut-être là où tu vas le plus loin dans ce style de plans: extérieurs non rattrapés, surexposés, où les détails disparaissent, et l'intérieur non rattrapé...

Là, il n'y a strictement rien, je pense même que la lumière qui était allumée pour la nuit était éteinte. Une chose que je fais toujours, même dans les téléfilms, et cela m'agace quand les gens ne comprennent pas, c'est que quand tu es comme ça [plan large à deux personnages dont l'ombre se découpe], tu as une silhouette, et quand tu vas au gros plan, c'est plus éclairé. Mais ça, dans la vie, c'est exactement ce qui se passe.

Donc là, c’est le soleil qui éclaire?

Oui, là c'est lumière du jour et voilà. Il n'y a rien à l'intérieur.

Et les murs, ils sont un peu plus clairs que les personnages...

Parce qu'ils étaient plus clairs.

Mais comment le mur se ré-éclaire?

C'est la lumière ambiante. A l'intérieur, il y avait peut-être une fenêtre que j'avais laissée un petit peu plus ouverte... Le mur doit être blanc et ça fait ce jaune orangé.

Et par terre ça réfléchit...

Oui. On le sent sur lui, sur le bras, parce que le
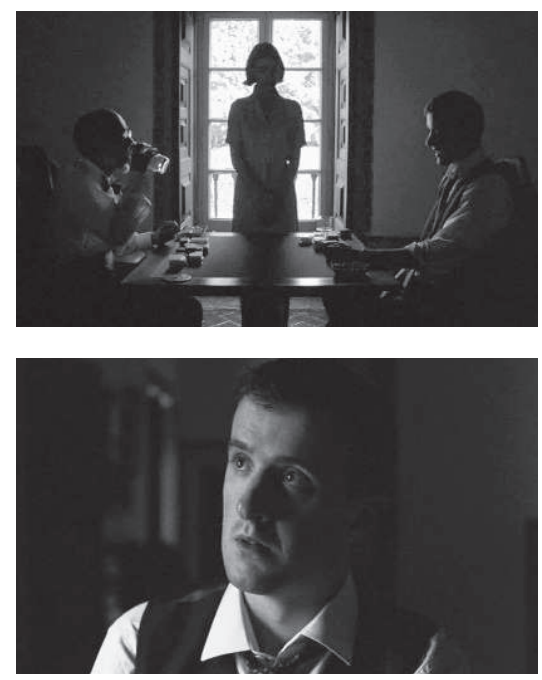

plancher, c'est des tomettes... Je pense que je ne pourrai plus faire des choses comme ça dans un film, ça ne se fait pas. Je ne pense pas pouvoir retrouver, au niveau de la réalisation de la photo, quelqu'un avec qui je puisse m'entendre comme avec lui. Parce qu'on était vraiment dans le même état d'esprit... Je pense même que je ne pourrai refaire de la photo que moimême pour moi. Je ne pourrais pas le faire avec quelqu'un d'autre, je ne trouverais plus... 